\title{
Stable Models of Fuzzy Propositional Formulas
}

\author{
Joohyung Lee and Yi Wang \\ School of Computing, Informatics, and Decision Systems Engineering \\ Arizona State University, Tempe, USA \\ $\{$ joolee, ywang 485$\}$ easu.edu
}

\begin{abstract}
We introduce the stable model semantics for fuzzy propositional formulas, which generalizes both fuzzy propositional logic and the stable model semantics of Boolean propositional formulas. Combining the advantages of both formalisms, the introduced language allows highly configurable default reasoning involving fuzzy truth values. We show that several properties of Boolean stable models are naturally extended to this formalism, and discuss how it is related to other approaches to combining fuzzy logic and the stable model semantics.
\end{abstract}

\section{Introduction}

Answer set programming (ASP) [1] is a widely applied declarative programming paradigm for the design and implementation of knowledge intensive applications. One of the attractive features of ASP is its capability to model the nonmonotonic aspect of knowledge. However, as its mathematical basis, the stable model semantics, is restricted to Boolean values, it is too rigid to represent imprecise and vague information. Fuzzy logic, as a form of many-valued logic, can handle vague information by interpreting propositions with a truth degree in the interval of real numbers $[0,1]$. The availability of various fuzzy operators gives the user great flexibility in combining truth degrees. However, the semantics of fuzzy logic is monotonic and is not flexible enough to handle default reasoning as allowed in answer set programming.

Both the stable model semantics and fuzzy logic are generalizations of classical propositional logic in different ways. While they do not subsume each other, it is clear that many real-world problems require both their strengths. This led to the body of work on combining fuzzy logic and the stable model semantics, known as fuzzy answer set programming (e.g., [2-9]). However, most work considers simple rule forms and do not allow connectives nested arbitrarily as in fuzzy logic.

Unlike existing work on fuzzy answer set semantics, in this paper, we extend the general stable model semantics from [10] to many-valued propositional formulas. The syntax of this language is the same as the syntax of fuzzy propositional logic. The semantics, on the other hand, distinguishes stable models from non-stable models. The language is a proper generalization of both fuzzy propositional logic and Boolean propositional formulas under the stable model semantics. This generalization is not simply a pure theoretical pursuit, but has practical use in conveniently modeling defaults involving fuzzy truth values in dynamic domains. For example, consider modeling dynamics of trust in social network. People trust each other in different degrees under some normal assumptions. If person $A$ trusts person $B$, then $A$ tends to trust person $C$ whom $B$ trusts to a degree which is positively correlated to the degree to which $A$ trusts $B$ and 
the degree to which $B$ trusts $C$. By default, the trust degrees would not change, but may decrease when a conflict arises between people. Modeling such a domain requires expressing defaults involving fuzzy truth values. We demonstrate that such examples can be conveniently modelled in our proposed language by taking advantage of its generality over the existing approaches to fuzzy ASP.

The paper is organized as follows. Section 2 reviews the syntax and the semantics of fuzzy propositional logic we discuss in the paper, as well as the stable model semantics of classical propositional formulas. Section 3 presents the stable model semantics of fuzzy propositional formulas along with examples, including the above trust example in the proposed language. Section 4 relates our fuzzy stable model semantics to the Boolean stable model semantics, and Section 5 relates it to other approaches to fuzzy ASP. Section 6 shows that several well-known properties of the Boolean stable model semantics can be easily extended to our fuzzy stable model semantics. Section 7 discusses other related work.

\section{Preliminaries}

\subsection{Review: Stable Models of Classical Propositional Formulas}

We review the definition of a stable model from [10] by limiting attention to the syntax of propositional formulas. Instead of defining stable models in terms of second-order logic as in [10], we express the same concept using auxiliary atoms that do not belong to the original signature. This slight reformulation will simplify our efforts in extending the stable model semantics to fuzzy propositional formulas without resorting to "secondorder fuzzy logic."

Let $\sigma$ be a classical propositional signature, let $\mathbf{p}=\left(p_{1}, \ldots, p_{n}\right)$ be a list of distinct atoms belonging to $\sigma$, and let $\mathbf{q}=\left(q_{1}, \ldots, q_{n}\right)$ be a list of new, distinct propositional atoms not belonging to $\sigma$. For two interpretations $I$ and $J$ of $\sigma$ that agree on all atoms in $\sigma \backslash \mathbf{p}, I \cup J_{\mathbf{q}}^{\mathbf{p}}$ denotes the interpretation of $\sigma \cup \mathbf{q}$ that

- agrees with $I$ on all atoms in $\sigma$, and

- for each atom $q_{i} \in \mathbf{q},\left(I \cup J_{\mathbf{q}}^{\mathbf{p}}\right)\left(q_{i}\right)=J\left(p_{i}\right){ }^{1}$

For any classical propositional formula $F$ of signature $\sigma, F^{*}(\mathbf{q})$ is a classical propositional formula of signature $\sigma \cup \mathbf{q}$ that is defined recursively as follows:

- $p_{i}^{*}=q_{i}$ for each $p_{i} \in \mathbf{p}$

- $F^{*}=F$ for any atom $F \notin \mathbf{p}$;

$-\perp^{*}=\perp ; \quad \top^{*}=\top$;

- $(\neg F)^{*}=\neg F$;

- $(F \wedge G)^{*}=F^{*} \wedge G^{*} ; \quad(F \vee G)^{*}=F^{*} \vee G^{*}$

- $(F \rightarrow G)^{*}=\left(F^{*} \rightarrow G^{*}\right) \wedge(F \rightarrow G)$.

Let $I$ and $J$ be two interpretations of $\sigma$, and let $\mathbf{p}$ be a subset of $\sigma$. We say $J \leq \mathbf{p} I$ if

- $J$ and $I$ agree on all atoms not in $\mathbf{p}$, and

- for all $p \in \mathbf{p}$, if $J \models p$, then $I \models p$.

\footnotetext{
${ }^{1} I(p)$ denotes the truth value of $p$ under $I$. We identify a list with a set if there is no confusion.
} 
We say $J<\mathbf{p} I$ if $J \leq \mathbf{p} I$ and $J \neq I$.

Definition 1. An interpretation $I$ is a stable model of $F$ relative to $\mathbf{p}$ (denoted $I \models$ $\mathrm{SM}[F ; \mathbf{p}])$

- if $I \models F$, and

- there is no interpretation $J$ such that $J<^{\mathbf{p}} I$ and $I \cup J_{\mathbf{q}}^{\mathbf{p}} \models F^{*}(\mathbf{q})$.

Example 1. Consider a logic program

$$
p \leftarrow \operatorname{not} q, \quad q \leftarrow \operatorname{not} p
$$

which is understood as an alternative notation for propositional formula $F_{1}=(\neg q \rightarrow$ $p) \wedge(\neg p \rightarrow q) . F_{1}^{*}(u, v)$ is $(\neg q \rightarrow u) \wedge(\neg q \rightarrow p) \wedge(\neg p \rightarrow v) \wedge(\neg p \rightarrow q)$. We check that $I_{1}=\{p\}$ (that is, $p$ is TRUE and $q$ is FALSE) ${ }^{2}$ is a stable model of $F_{1}$ (relative to $\{p, q\}): I_{1}$ satisfies $F_{1}$, and $\emptyset$ is the only interpretation $J$ such that $J<{ }^{p q} I_{1}$. However, $I_{1} \cup J_{u v}^{p q}=\{p\}$ does not satisfy $F_{1}^{*}(u, v)$ because it does not satisfy the first conjunctive term of $F_{1}^{*}(u, v)$. Similarly, we can check that $\{q\}$ is another stable model of $F_{1}$.

\subsection{Review: Fuzzy Logic}

Let $\sigma$ be a fuzzy propositional signature, which is a set of symbols called fuzzy atoms. In addition, we assume the presence of a set $\mathbb{C}$ of fuzzy conjunction symbols, a set $\mathbb{D}$ of fuzzy disjunction symbols, a set $\mathbb{N}$ of fuzzy negation symbols, and a set $\mathbb{I}$ of fuzzy implication symbols.

A fuzzy (propositional) formula of $\sigma$ is defined recursively as follows.

- every fuzzy atom $p \in \sigma$ is a fuzzy formula;

- every numeric constant $\bar{c}$ where $c$ is a real number in $[0,1]$ is a fuzzy formula;

- if $F$ is a fuzzy formula, then $\neg F$ is a fuzzy formula, where $\neg \in \mathbb{N}$;

- if $F$ and $G$ are fuzzy formulas, then $F \otimes G, F \oplus G$ and $F \rightarrow G$ are fuzzy formulas, where $\otimes \in \mathbb{C}, \oplus \in \mathbb{D}$, and $\rightarrow \in \mathbb{I}$.

The models of a fuzzy formula are defined as follows [11]. The fuzzy truth values are the real numbers in the range $[0,1]$. A fuzzy interpretation $I$ of $\sigma$ is a mapping from $\sigma$ into $[0,1]$.

The fuzzy operators are functions mapping one or a pair of truth values into a truth value. Among the operators, $\neg$ denotes a function from $[0,1]$ into $[0,1] ; \otimes, \oplus$, and $\rightarrow$ denote functions from $[0,1] \times[0,1]$ into $[0,1]$. The actual mapping performed by each operator can be defined in many different ways, but all of them satisfy the following conditions, which imply that the operators are generalizations of the corresponding classical propositional connectives: ${ }^{3}$

- a fuzzy negation $\neg$ is decreasing, and satisfies $\neg(0)=1$ and $\neg(1)=0$;

\footnotetext{
${ }^{2}$ We identify a propositional interpretation with the set of atoms that are true in it.

${ }^{3}$ We say that a function $f$ of arity $n$ is increasing in its $i$-th argument $(1 \leq i \leq n)$ if $f\left(\arg _{1}, \ldots, \arg _{i}, \ldots, \arg _{n}\right) \leq f\left(\arg _{1}, \ldots, \arg _{i}^{\prime}, \ldots, \arg _{n}\right)$ for all arguments such that $\arg _{i} \leq \arg _{i}^{\prime}$; $f$ is said to be increasing if it is increasing in all its arguments. The definition of decreasing is similar.
} 
- a fuzzy conjunction $\otimes$ is increasing, commutative, associative, and $\otimes(1, x)=x$ for all $x \in[0,1]$;

- a fuzzy disjunction $\oplus$ is increasing, commutative, associative, and $\oplus(0, x)=x$ for all $x \in[0,1]$;

- a fuzzy implication $\rightarrow$ is decreasing in its first argument and increasing in its second argument; and $\rightarrow(1, x)=x$ and $\rightarrow(0,0)=1$ for all $x \in[0,1]$.

Figure 1 lists some specific fuzzy operators that we use in this paper.

\begin{tabular}{|c|c|c|}
\hline Symbol & Name & Definition \\
\hline$\otimes_{l}$ & Łukasiewicz t-norm & $\otimes_{l}(x, y)=\max (x+y-1,0)$ \\
\hline$\oplus_{l}$ & Łukasiewicz t-conorm & $\oplus_{l}(x, y)=\min (x+y, 1)$ \\
\hline$\otimes_{m}$ & minimum t-norm & $\otimes_{m}(x, y)=\min (x, y)$ \\
\hline$\oplus_{m}$ & maximum t-conorm & $\oplus_{m}(x, y)=\max (x, y)$ \\
\hline$\otimes_{p}$ & product t-norm & $\otimes_{p}(x, y)=x \cdot y$ \\
\hline$\oplus_{p}$ & product t-conorm & $\oplus_{p}(x, y)=x+y-x \cdot y$ \\
\hline$\neg_{s}$ & standard negator & $\neg_{s}(x)=1-x$ \\
\hline$\rightarrow_{r}$ & the residual implicator of $\otimes_{m}$ & $\rightarrow_{r}(x, y)= \begin{cases}1 & \text { if } x \leq y \\
y & \text { otherwise }\end{cases}$ \\
\hline$\rightarrow_{s}$ & the S-implicator induced by $\neg_{s}$ and $\oplus_{m}$ & $\rightarrow_{s}(x, y)=\max (1-x, y)$ \\
\hline
\end{tabular}

Fig. 1. Some t-norms, t-conorms, negator, and implicators

The truth value of a fuzzy formula $F$ under $I$, denoted $F^{I}$, is defined recursively as follows:

- for any atom $p \in \sigma, p^{I}=I(p)$;

- for any numeric constant $\bar{c}, \bar{c}^{I}=c$;

- $(\neg F)^{I}=\neg\left(F^{I}\right)$;

- $(F \otimes G)^{I}=\otimes\left(F^{I}, G^{I}\right) ;(F \oplus G)^{I}=\oplus\left(F^{I}, G^{I}\right) ;(F \rightarrow G)^{I}=\rightarrow\left(F^{I}, G^{I}\right)$.

(For simplicity, we identify the symbols for the fuzzy operators with the truth value functions represented by them.)

Definition 2. We say that a fuzzy interpretation $I$ satisfies a fuzzy formula $F$ w.r.t. a threshold $y \in[0,1]$ if $F^{I} \geq y$, and denote it by $I={ }_{y} F$. We call $I$ a fuzzy $y$-model of $F$.

We often omit the threshold $y$ when it is 1 .

\section{Definition and Examples}

We extend the notion of $J<^{\mathbf{p}} I$ in Section 2.1 as follows. For any two fuzzy interpretations $J$ and $I$ of the same signature $\sigma$ and any subset $\mathbf{p}$ of $\sigma$, we say $J \leq^{\mathbf{p}} I$ if

- $J$ and $I$ agree on all fuzzy atoms not in $\mathbf{p}$, and

- for all $p \in \mathbf{p}, p^{J} \leq p^{I}$.

We say $J<^{\mathbf{p}} I$ if $J \leq^{\mathbf{p}} I$ and $J \neq I$.

As before, we assume a list $\mathbf{q}=\left(q_{1}, \ldots, q_{n}\right)$ of new, distinct fuzzy atoms that corresponds to $\mathbf{p}=\left(p_{1}, \ldots, p_{n}\right)$, and define $I \cup J_{\mathbf{q}}^{\mathbf{p}}$ in the same way. That is, when $I$ and $J$ agree on all atoms in $\sigma \backslash \mathbf{p}, I \cup J_{\mathbf{q}}^{\mathbf{p}}$ denotes the interpretation of $\sigma \cup \mathbf{q}$ that 
- agrees with $I$ on all atoms in $\sigma$, and

- for each $q_{i} \in \mathbf{q},\left(I \cup J_{\mathbf{q}}^{\mathbf{p}}\right)\left(q_{i}\right)=J\left(p_{i}\right)$.

The definition of $F^{*}$ is also extended in a straightforward way: For any fuzzy formula $F$ of signature $\sigma, F^{*}(\mathbf{q})$ is defined as follows.

- $p_{i}^{*}=q_{i}$ for each $p_{i} \in \mathbf{p}$;

- $F^{*}=F$ for any atom $F \notin \mathbf{p}$;

- $\bar{c}^{*}=\bar{c}$ for any numeric constant $\bar{c}$;

- $(\neg F)^{*}=\neg F$;

- $(F \otimes G)^{*}=F^{*} \otimes G^{*} ; \quad(F \oplus G)^{*}=F^{*} \oplus G^{*} ;$

- $(F \rightarrow G)^{*}=\left(F^{*} \rightarrow G^{*}\right) \otimes_{m}(F \rightarrow G){ }^{4}$

Definition 3. A fuzzy interpretation $I$ is a fuzzy y-stable model of $F$ relative to $\mathbf{p}$ (denoted $\left.I={ }_{y} \mathrm{SM}[F ; \mathbf{p}]\right)$ if

- $I \models_{y} F$, and

- there is no fuzzy interpretation $J$ such that $J<\mathbf{p} I$ and $I \cup J_{\mathbf{q}}^{\mathbf{p}} \models{ }_{y} F^{*}(\mathbf{q})$.

We often omit the threshold $y$ when it is 1 , and omit $\mathbf{p}$ if it contains all atoms in $\sigma$.

Clearly, when $\mathbf{p}$ is empty, Definition 3 reduces to the definition of a fuzzy model in Definition 2 because there is no $J$ such that $J<^{\emptyset} I$.

Also, Definition 3 is very similar to the definition of a stable model for classical propositional formulas in Definition 1. The main difference is that simply in the latter, atoms may have various degrees of truth, and accordingly the notion of $J<\mathbf{p} I$ is more general. The precise relationship between the definitions is discussed in Section 4.

Example 2. Consider the fuzzy formula $F=\neg_{s} p \rightarrow_{r} q$ and the interpretation $I=$ $\{(p, 0),(q, 0.6)\} \cdot F^{*}(u, v)$ is

$$
\left(\left(\neg_{s} p\right)^{*} \rightarrow_{r} q^{*}\right) \otimes_{m}\left(\neg_{s} p \rightarrow_{r} q\right)=\left(\neg_{s} p \rightarrow_{r} v\right) \otimes_{m}\left(\neg_{s} p \rightarrow_{r} q\right) .
$$

$I \models_{0.6} \mathrm{SM}[F ; p, q]$. First, it is easy to see that $I \models_{0.6} F$, as

$$
F^{I}=\rightarrow_{r}\left(\left(\neg_{s} p\right)^{I}, q^{I}\right)=\rightarrow_{r}\left(1-p^{I}, q^{I}\right)=\rightarrow_{r}(1,0.6)=0.6 .
$$

Suppose there exists $J<{ }^{p q} I$ such that $I \cup J_{u v}^{p q} \models_{0.6} F$, i.e.,

$$
\begin{aligned}
F^{*}(u, v)^{I \cup J_{u v}^{p q}} & =\min \left(\rightarrow_{r}\left(\neg_{s}\left(p^{I}\right), v^{I \cup J_{u v}^{p q}}\right), \rightarrow_{r}\left(\neg_{s}\left(p^{I}\right), q^{I}\right)\right) \\
& =\min \left(\rightarrow_{r}\left(1, q^{J}\right), 0.6\right) \\
& =\min \left(q^{J}, 0.6\right) \geq 0.6 .
\end{aligned}
$$

So $q^{J} \geq 0.6$. This contradicts the assumption that $J<^{p q} I$. Therefore, such $J$ does not exist, and $I$ is a 0.6 -stable model of $F$.

\footnotetext{
${ }^{4}$ Note the use of $\otimes_{m}$ here; the value of "conjunction" of $\left(F^{*} \rightarrow G^{*}\right)$ and $(F \rightarrow G)$ needs not be smaller than the value of $\left(F^{*} \rightarrow G^{*}\right)$ and the value of $(F \rightarrow G)$. It turns out that $\otimes_{m}$ is the only t-norm that satisfies this property.
} 
Example 3. $p$ and $\left.\urcorner_{s}\right\urcorner_{s} p$ have the same fuzzy models, but their stable models are different. This is similar to the fact that $p$ and $\neg \neg p$ have different stable models according to the semantics from [10].

Clearly, any interpretation $I=\{(p, y)\}$, where $y$ is any positive real number in $[0,1]$, is a $y$-stable model of $p$ relative to $\{p\}$. On the other hand, $I=\{(p, y)\}$ is not a $y$-stable model of $\left.F=\neg_{s}\right\urcorner_{s} p$ relative to $\{p\}$. Formula $F^{*}(u)$ is $\left.\neg_{s}\right\urcorner_{s} F$, and although $I \models_{y} F$, we have $I \cup J_{u}^{p} \models_{y} F^{*}(u)$ regardless of any $J$.

Example 4. Let $F_{1}=p \rightarrow_{s} p$ and $F_{2}=\neg_{s} p \oplus_{m} p$. Their fuzzy models are the same, but their stable models are not. This is similar to the relation between $p \rightarrow p$ and $\neg p \vee p$ in the Boolean stable model semantics. Indeed, observe that $F_{1}^{*}(u)=\left(p \rightarrow_{s} p\right) \otimes_{m}\left(u \rightarrow_{s} u\right)$ and $F_{2}^{*}(u)=\neg_{s} p \oplus_{m} u$.

The interpretation $I=\{(p, 1)\}$ is not a 1-stable model of $F_{1}$ relative to $p$, as witnessed by $J=\{(p, 0)\}$. However, $I$ is a 1-stable model of $F_{2}$ relative to $p$ : for any $J$,

$$
F_{2}^{*}(u)^{I \cup J_{u}^{p}}=\max \left(1-p^{I}, p^{J}\right)=\max \left(0, p^{J}\right)=p^{J} .
$$

So, for $I \cup J_{u}^{p}$ to satisfy $F_{2}^{*}(u)$ to degree $1, p^{J}$ should be 1 . Consequently, it is not possible to have $J<^{p} I$.

The following example illustrates how the commonsense law of inertia involving fuzzy truth values can be represented.

Example 5. Let $\sigma$ be $\{p, n p, q, n q\}$ and let $F$ be $F_{1} \otimes_{m} F_{2}$, where $F_{1}$ represents that $p$ and $n p$ are complementary, i.e., the sum of their truth values is 1 :

$$
F_{1}=\neg_{s}\left(p \otimes_{l} n p\right) \otimes_{m} \neg_{s} \neg_{s}\left(p \oplus_{l} n p\right) .
$$

$F_{2}$ represents that by default $p$ has the truth value of $q$, and $n p$ has the truth value of $n q$ :

$$
\left.\left.F_{2}=\left(\left(q \otimes_{m} \neg_{s}\right\urcorner_{s} p\right) \rightarrow_{r} p\right) \otimes_{m}\left(\left(n q \otimes_{m} \neg_{s}\right\urcorner_{s} n p\right) \rightarrow_{r} n p\right) .
$$

Let $\mathbf{p}=\{p, n p\}$ and $\mathbf{u}=\{u, n u\} . F^{*}(\mathbf{u})$ is

$$
\begin{aligned}
& \left.\left.\neg_{s}\left(p \otimes_{l} n p\right) \otimes_{m}\right\urcorner_{s}\right\urcorner_{s}\left(p \oplus_{l} n p\right) \\
& \left.\left.\left.\left.\otimes_{m}\left(\left(q \otimes_{m}\right\urcorner_{s}\right\urcorner_{s} p\right) \rightarrow_{r} u\right) \otimes_{m}\left(\left(q \otimes_{m}\right\urcorner_{s}\right\urcorner_{s} p\right) \rightarrow_{r} p\right) \\
& \left.\left.\left.\left.\otimes_{m}\left(\left(n q \otimes_{m}\right\urcorner_{s}\right\urcorner_{s} n p\right) \rightarrow_{r} n u\right) \otimes_{m}\left(\left(n q \otimes_{m}\right\urcorner_{s}\right\urcorner_{s} n p\right) \rightarrow_{r} n p\right) .
\end{aligned}
$$

One can check that interpretation $I_{1}=\{(p, x),(n p, 1-x),(q, x),(n q, 1-x)\}$ ( $x$ is any value in $[0,1])$ is a 1 -stable model of $F$ relative to $(p, n p)$; interpretation $I_{2}=$ $\{(p, y),(n p, 1-y),(q, x),(n q, 1-x)\}$, where $y \neq x$, is not.

On the other hand, if we conjoin $F$ with $\left(\bar{y} \rightarrow_{r} p\right) \otimes_{m}\left(\overline{1-y} \rightarrow_{r} n p\right)$, the default behavior is overridden: $I_{1}$ is not a 1-stable model of $F \otimes_{m}\left(\bar{y} \rightarrow_{r} p\right) \otimes_{m}\left(\overline{1-y} \rightarrow_{r} n p\right)$ relative to $(p, n p)$, but $I_{2}$ is.

This behavior is useful in expressing the commonsense law of inertia involving fuzzy values. Suppose $q$ represents some fluent at time $t$, and $p$ represents the fluent at time $t+1$. Then $F$ states that, "by default, the fluent retains the previous value." The default value is overridden if there is an action that sets $p$ to a different value. 
Example 6. The trust example in the introduction can be formalized in the fuzzy stable model semantics as follows. Below $x, y, z$ are schematic variables ranging over people, and $t$ is a schematic variable ranging over time steps. Trust $(x, y, t)$ is a fuzzy atom representing that " $x$ trusts $y$ at time $t$." Similarly, Distrust $(x, y, t)$ is a fuzzy atom representing that " $x$ distrusts $y$ at time $t$."

The trust relation is reflexive:

$$
F_{1}=\operatorname{Trust}(x, x, t) .
$$

The trust and distrust degrees are complementary, i.e., their sum is 1 (similar to Example 5):

$$
\begin{aligned}
& F_{2}=\neg_{s}\left(\operatorname{Trust}(x, y, t) \otimes_{l} \operatorname{Distrust}(x, y, t)\right), \\
& \left.F_{3}=\neg_{s}\right\urcorner_{s}\left(\operatorname{Trust}(x, y, t) \oplus_{l} \operatorname{Distrust}(x, y, t)\right) .
\end{aligned}
$$

Initially, if $x$ trusts $y$ to degree $d_{1}$ and $y$ trusts $z$ to degree $d_{2}$, then $x$ trusts $z$ to degree $d_{1} \times d_{2}$; further the initial distrust degree is 1 minus the initial trust degree.

$$
\begin{aligned}
& F_{4}=\operatorname{Trust}(x, y, 0) \otimes_{p} \operatorname{Trust}(y, z, 0) \rightarrow_{r} \operatorname{Trust}(x, z, 0), \\
& F_{5}=\neg_{s} \operatorname{Trust}(x, y, 0) \rightarrow_{r} \operatorname{Distrust}(x, y, 0) .
\end{aligned}
$$

The inertia assumption (similar to Example 5):

$$
\begin{aligned}
& \left.\left.F_{6}=\operatorname{Trust}(x, y, t) \otimes_{m}\right\urcorner_{s}\right\urcorner_{s} \operatorname{Trust}(x, y, t+1) \rightarrow_{r} \operatorname{Trust}(x, y, t+1), \\
& \left.\left.F_{7}=\operatorname{Distrust}(x, y, t) \otimes_{m}\right\urcorner_{s}\right\urcorner_{s} \operatorname{Distrust}(x, y, t+1) \rightarrow_{r} \operatorname{Distrust}(x, y, t+1) .
\end{aligned}
$$

A conflict increases the distrust degree by the conflict degree:

$$
\begin{aligned}
& F_{8}=\operatorname{Conflict}(x, y, t) \oplus_{l} \operatorname{Distrust}(x, y, t) \rightarrow_{r} \text { Distrust }(x, y, t+1), \\
& F_{9}=\neg_{s}\left(\text { Conflict }(x, y, t) \oplus_{l} \operatorname{Distrust}(x, y, t)\right) \rightarrow_{r} \text { Trust }(x, y, t+1) .
\end{aligned}
$$

Let $F_{T W}$ be $F_{1} \otimes_{m} F_{2} \otimes_{m} \cdots \otimes_{m} F_{9}$. Suppose we have the formula $F_{F a c t}=$ Fact $_{1} \otimes_{m}$ Fact $_{2}$ that gives the initial trust degree.

$$
\begin{aligned}
& \text { Fact }_{1}=\overline{0.8} \rightarrow_{r} \text { Trust }(\text { Alice }, \text { Bob }, 0), \\
& \text { Fact }_{2}=\overline{0.7} \rightarrow_{r} \text { Trust }(\text { Bob }, \text { Carol }, 0) .
\end{aligned}
$$

Although there is no fact about how much Alice trusts Carol, any 1-stable model of $F_{T W} \otimes_{m} F_{\text {Fact }}$ assigns value 0.56 to the atom Trust(Alice, Carol, 0 ). On the other hand, the 1-stable model assigns value 0 to Trust(Alice,David, 0$)$ due to the closed world assumption under the stable model semantics.

When we conjoin $F_{T W} \otimes F_{F a c t}$ with $\overline{0.2} \rightarrow$ Conflict(Alice,Carol,0), the 1-stable model of $F_{T W} \otimes_{m} F_{\text {Fact }} \otimes_{m}(\overline{0.2} \rightarrow$ Conflict(Alice, Carol, 0$\left.)\right)$ manifests that the trust degree between Alice and Carol decreases to 0.36 at time 1. More generally, if we have more actions that change the trust degree in various ways, by specifying the entire history of actions, we can determine the evolution of the trust distribution among all the participants. Useful decisions can be made based on this information. For example, Alice may decide not to share her personal pictures to those whom she trusts less than degree 0.48 .

Note that this example, like Example 5, uses nested connectives, such as $\neg_{s} \neg s$, that are not available in previous fuzzy ASP semantics, such as [2,3]. 


\section{Relation to Boolean-Valued Stable Models}

The Boolean stable model semantics in Section 2.1 can be embedded into the fuzzy stable model semantics as follows:

For any classical propositional formula $F$, define $F^{\text {fuzzy }}$ to be the fuzzy propositional formula obtained from $F$ by replacing $\perp$ with $\overline{0}, \top$ with $\overline{1}, \neg$ with $\neg_{s}, \wedge$ with $\otimes_{m}, \vee$ with $\oplus_{m}$, and $\rightarrow$ with $\rightarrow_{s}$. We identify the signature of $F^{f u z z y}$ with the signature of $F$. Also, for any interpretation $I$, we define the corresponding fuzzy interpretation $I^{f u z z y}$ as

- $I^{\text {fuzzy }}(p)=1$ if $I(p)=$ TRUE;

- $I^{\text {fuzzy }}(p)=0$ otherwise.

The following theorem tells us that the Boolean-valued stable model semantics can be viewed as a special case of the fuzzy stable model semantics.

Theorem 1 For any classical propositional formula $F$ and any classical propositional interpretation $I, I$ is a stable model of $F$ relative to $\mathbf{p}$ iff $I^{\text {fuzzy }}$ is a 1 -stable model of $F^{\text {fuzzy }}$ relative to $\mathbf{p}$.

Example 7. Let $F$ be the classical propositional formula $\neg p \rightarrow q$. $F$ has only one stable model $I=\{q\}$. Clearly $I^{\text {fuzzy }}=\{(p, 0),(q, 1)\}$ is a 1-stable model of $F^{\text {fuzzy }}=\neg_{s} p \rightarrow_{s}$ $q$.

Theorem 1 does not hold for an arbitrary choice of operators, as illustrated by the following example.

Example 8. Let $F$ be the classical propositional formula $p \vee p$. Classical interpretation $I=\{p\}$ is a stable model of $F$. However, $I^{\text {fuzzy }}=\{(p, 1)\}$ is not a stable model of $F^{\prime}=p \oplus_{l} p$ because there is $J=\{(p, 0.5)\}$ such that $I \cup J_{q}^{p} \models_{1} q \oplus_{l} q$.

However, one direction of Theorem 1 holds for arbitrary choice of fuzzy operators.

Theorem 2 For any classical propositional formula $F$, let $F_{1}^{f u z z y}$ be the fuzzy formula obtained from $F$ by replacing $\perp$ with $\overline{0}, \top$ with $\overline{1}, \neg$ with any fuzzy negation symbol, $\wedge$ with any fuzzy conjunction symbol, $\vee$ with any fuzzy disjunction symbol, and $\rightarrow$ with any fuzzy implication symbol. For any classical propositional interpretation $I$, if $I^{\text {fuzzy }}$ is a 1 -stable model of $F_{1}^{\text {fuzzy }}$ relative to $\mathbf{p}$, then $I$ is a stable model of $F$ relative to $\mathbf{p}$.

\section{Relation to Other Approaches to Fuzzy ASP}

\subsection{Relation to Stable Models of Normal FASP Programs}

A normal FASP program is a finite set of rules of the form

$$
a \leftarrow b_{1} \otimes \ldots \otimes b_{m} \otimes \neg b_{m+1} \otimes \ldots \otimes \neg b_{n},
$$

where $n \geq m \geq 0, a, b_{1}, \ldots, b_{n}$ are fuzzy atoms or numeric constants in $[0,1]$, and $\otimes$ is any fuzzy conjunction. We identify the rule with the fuzzy implication

$$
b_{1} \otimes \ldots \otimes b_{m} \otimes \neg_{s} b_{m+1} \otimes \ldots \otimes \neg_{s} b_{n} \rightarrow_{r} a .
$$


We say that a fuzzy interpretation $I$ of signature $\sigma$ satisfies a rule $R$ if $R^{I}=1$. I satisfies an FASP program $\Pi$ if $I$ satisfies every rule in $\Pi$.

According to [2], an interpretation $I$ is a fuzzy answer set of a normal FASP program $\Pi$ if $I$ satisfies $\Pi$, and no interpretation $J$ such that $J<^{\sigma} I$ satisfies the reduct of $\Pi$ w.r.t. $I$, which is the program obtained from $\Pi$ by replacing each negative literal $\neg b$ with the constant for $1-b^{I}$.

Theorem 3 For any normal FASP program $\Pi=\left\{r_{1}, \ldots, r_{n}\right\}$, let $F$ be the fuzzy formula $r_{1} \otimes_{m} \ldots \otimes_{m} r_{n}$. An interpretation I is a fuzzy answer set of $\Pi$ in the sense of [2] if and only if $I$ is a 1-stable model of $F$.

Example 9. Let $\Pi$ be the following program

$$
p \leftarrow \neg q, \quad q \leftarrow \neg p .
$$

The answer sets of $\Pi$ according to [2] are $\{(p, x),(q, 1-x)\}$, where $x$ is any value in $[0,1]$ : the corresponding fuzzy formula $F$ is $\left(\neg_{s} q \rightarrow_{r} p\right) \otimes_{m}\left(\neg_{s} p \rightarrow_{r} q\right) ; F^{*}(u, v)$ is

$$
F \otimes_{m}\left(\left(\neg_{s} q \rightarrow_{r} u\right) \otimes_{m}\left(\neg_{s} p \rightarrow_{r} v\right)\right) .
$$

One can check that the 1 -stable models of $F$ are also $\{(p, x),(q, 1-x)\}$, where $x \in[0,1]$.

\subsection{Relation to Fuzzy Equilibrium Logic}

Like the fuzzy stable model semantics introduced in this paper, fuzzy equilibrium logic [12] generalizes fuzzy ASP programs to arbitrary propositional formulas, but its definition is quite complex as it is based on a pair of intervals and considers strong negation as one of the primary connectives. Nonetheless we show that fuzzy equilibrium logic is essentially equivalent to the fuzzy stable model semantics where the threshold is restricted to 1 and all atoms are subject to minimization.

Review: Fuzzy Equilibrium Logic We first review the definition of fuzzy equilibrium logic from [12]. The syntax is the same as the one we reviewed in Section 2.2 except that a new connective $\sim$ (strong negation) may appear in front of atoms. ${ }^{5}$ For any fuzzy propositional signature $\sigma$, a (fuzzy N5) valuation is a mapping from $\{h, t\} \times \sigma$ to subintervals of $[0,1]$ such that $V(t, a) \subseteq V(h, a)$ for each atom $a \in \sigma$. For $V(w, a)=[u, v]$, where $w \in\{h, t\}$, we write $V^{-}(w, a)$ to denote the lower bound $u$ and $V^{+}(w, a)$ to denote the upper bound $v$. The truth value of a fuzzy formula under $V$ is defined as follows.

- $V(w, \bar{c})=[c, c]$ for any numeric constant $\bar{c}$;

- $V(w, \sim a)=\left[1-V^{+}(w, a), 1-V^{-}(w, a)\right]$, where $\sim$ is the symbol for strong negation;

- $V(w, F \otimes G)=\left[V^{-}(w, F) \otimes V^{-}(w, G), V^{+}(w, F) \otimes V^{+}(w, G)\right] ;{ }^{6}$

- $V(w, F \oplus G)=\left[V^{-}(w, F) \oplus V^{-}(w, G), V^{+}(w, F) \oplus V^{+}(w, G)\right]$;

- $V(h, \neg F)=\left[1-V^{-}(t, F), 1-V^{-}(h, F)\right]$;

- $V(t, \neg F)=\left[1-V^{-}(t, F), 1-V^{-}(t, F)\right]$;

\footnotetext{
${ }^{5}$ The definition from [12] allows strong negation in front of any formulas. We restrict its occurrence only in front of atoms as usual in answer set programs.

${ }^{6}$ For readability, we write the infix notation $(x \odot y)$ in place of $\odot(x, y)$.
} 


$$
\begin{gathered}
-V(h, F \rightarrow G)=\left[\min \left(V^{-}(h, F) \rightarrow V^{-}(h, G), V^{-}(t, F) \rightarrow V^{-}(t, G)\right),\right. \\
\left.\quad V^{-}(h, F) \rightarrow V^{+}(h, G)\right] ; \\
\text { - } V(t, F \rightarrow G)=\left[V^{-}(t, F) \rightarrow V^{-}(t, G), V^{-}(t, F) \rightarrow V^{+}(t, G)\right] .
\end{gathered}
$$

A valuation $V$ is a (fuzzy N5) model of a formula $F$ if $V^{-}(h, F)=1$, which implies $V^{+}(h, F)=V^{-}(t, F)=V^{+}(t, F)=1$. For two valuations $V$ and $V^{\prime}$, we say $V^{\prime} \preceq V$ if $V^{\prime}(t, a)=V(t, a)$ and $V(h, a) \subseteq V^{\prime}(h, a)$ for all atoms $a$. We say $V^{\prime} \prec V$ if $V^{\prime} \preceq V$ and $V^{\prime} \neq V$. We say that a model $V$ of $F$ is $h$-minimal if there is no model $V^{\prime}$ of $F$ such that $V^{\prime} \prec V$. An h-minimal fuzzy N5 model $V$ of $F$ is a fuzzy equilibrium model of $F$ if $V(h, a)=V(t, a)$ for all atoms $a$.

In the Absence of Strong Negation We first establish the correspondence between fuzzy stable models and fuzzy equilibrium models in the absence of strong negation. As in [12], we assume that the fuzzy negation $\neg$ is $\urcorner_{s}$.

For any valuation $V$, we define a fuzzy interpretation $I_{V}$ as $p^{I_{V}}=V^{-}(h, p)$ for each atom $p \in \sigma$.

Theorem 4 Let $F$ be a fuzzy propositional formula of $\sigma$ that contains no strong negation.

(a) A valuation $V$ of $\sigma$ is a fuzzy equilibrium model of $F$ iff $V^{-}(h, p)=V^{-}(t, p)$, $V^{+}(h, p)=V^{+}(t, p)=1$ for all atoms $p$ in $\sigma$ and $I_{V}$ is a 1-stable model of $F$ relative to $\sigma$.

(b) An interpretation $I$ of $\sigma$ is a 1-stable model of $F$ relative to $\sigma$ iff $I=I_{V}$ for some fuzzy equilibrium model $V$ of $F$.

In the Presence of Strong Negation In this section we extend the relationship between fuzzy equilibrium logic and our stable model semantics by allowing strong negation. This is done by simulating strong negation by new atoms in our semantics.

Let $\sigma$ denote the signature. For a fuzzy formula $F$ over $\sigma$ that may contain strong negation, define $F^{\prime}$ over $\sigma \cup\{n p \mid p \in \sigma\}$ as the formula obtained from $F$ by replacing all strong negations of atom $\sim p$ with a new atom $n p$. The transformation nneg $(F)$ ("no strong negation") is defined as $\left.n n e g(F)=F^{\prime} \otimes_{m} \bigotimes_{p \in \sigma}\right\urcorner_{s}\left(p \otimes_{l} n p\right)$.

For any valuation $V$ of $\sigma$, we define the interpretation $I_{V}$ of $\sigma \cup\{n p \mid p \in \sigma\}$ as

$$
\begin{cases}p^{I_{V}}=V^{-}(h, p) & \text { for each } p \in \sigma ; \\ n p^{I_{V}}=1-V^{+}(h, p) & \text { for each } n p \notin \sigma .\end{cases}
$$

Theorem 5 For any fuzzy formula $F$ of signature $\sigma$ that may contain strong negation,

(a) A valuation $V$ of $\sigma$ is a fuzzy equilibrium model of $F$ iff $V(h, p)=V(t, p)$ for all atoms $p$ in $\sigma$ and $I_{V}$ is a 1-stable model of nneg $(F)$ relative to $\sigma \cup\{n p \mid p \in \sigma\}$.

(b) An interpretation I of $\sigma \cup\{n p \mid p \in \sigma\}$ is a 1-stable model of nneg $(F)$ relative to $\sigma \cup\{n p \mid p \in \sigma\}$ iff $I=I_{V}$ for some fuzzy equilibrium model $V$ of $F$.

Example 10. For fuzzy formula $F=\left(\overline{0.2} \rightarrow_{r} p\right) \otimes_{m}\left(\overline{0.3} \rightarrow_{r} n p\right)$, formula $n n e g(F)$ is

$$
\left(\overline{0.2} \rightarrow_{r} p\right) \otimes_{m}\left(\overline{0.3} \rightarrow_{r} n p\right) \otimes_{m} \neg_{s}\left(p \otimes_{l} n p\right) .
$$

One can check that the valuation $V$ defined as $V(w, p)=[0.2,0.7]$ is the only equilibrium model of $F$, and the interpretation $I_{V}=\{(p, 0.2),(n p, 0.3)\}$ is the only 1-stable model of $\operatorname{nneg}(F)$. 
This idea of eliminating strong negation in favor of new atoms was used in Examples 5 and 6.

\section{Properties of Fuzzy Stable Models}

In this section, we show that several well-known properties of the Boolean stable model semantics can be naturally extended to the fuzzy stable model semantics.

\subsection{Alternative Definition of $F^{*}$}

Proposition 1 For any fuzzy formulas $F, G$ and any fuzzy interpretations $I, J$ such that $J \leq \mathbf{p} I$,

- $I \cup J_{\mathbf{q}}^{\mathbf{p}} \models_{y} \neg F^{*}(\mathbf{q}) \otimes_{m} \neg F \quad$ iff $I \cup J_{\mathbf{q}}^{\mathbf{p}} \models_{y} \neg F$;

$-I \cup J_{\mathbf{q}}^{\mathbf{p}} \models_{y}\left(F^{*} \otimes G^{*}\right)(\mathbf{q}) \otimes_{m}(F \otimes G)$ iff $I \cup J_{\mathbf{q}}^{\mathbf{p}} \models_{y}\left(F^{*} \otimes G^{*}\right)(\mathbf{q})$;

- $I \cup J_{\mathbf{q}}^{\mathbf{p}} \models_{y}\left(F^{*} \oplus G^{*}\right)(\mathbf{q}) \otimes_{m}(F \oplus G) \quad$ iff $I \cup J_{\mathbf{q}}^{\mathbf{p}} \models_{y}\left(F^{*} \oplus G^{*}\right)(\mathbf{q})$.

This proposition tells us that $F^{*}$ in Section 3 can be equivalently defined by treating the fuzzy operators in the uniform way without affecting stable models.

- $(\neg F)^{*}=\neg F^{*} \otimes_{m} \neg F$;

- $(F \odot G)^{*}=\left(F^{*} \odot G^{*}\right) \otimes_{m}(F \odot G)$ for any binary operator $\odot$.

\subsection{Theorem on Constraints}

In answer set programming, constraints-rules with $\perp$ in the head-play an important role in view of the fact that adding a constraint eliminates the stable models that "violate" the constraint. The following theorem is the counterpart of Theorem 3 from [10] for fuzzy propositional formulas.

Theorem 6 For any fuzzy formulas $F$ and $G, I$ is a 1-stable model of $F \otimes \neg G$ (relative to $\mathbf{p}$ ) if and only if $I$ is a 1-stable model of $F$ (relative to $\mathbf{p}$ ) and $I \models_{1} \neg G$.

Example 11. Consider $F=\left(\neg_{s} p \rightarrow_{r} q\right) \otimes_{m}\left(\neg_{s} q \rightarrow_{r} p\right) \otimes_{m} \neg_{s} p$. Formula $F$ has only one 1-stable model $I=\{(p, 0),(q, 1)\}$, which is the only 1-stable model of $\left(\neg_{s} p \rightarrow_{r} q\right) \otimes_{m}\left(\neg_{s} q \rightarrow_{r} p\right)$ that satisfies $\neg_{s} p$ to degree 1 .

If we consider a more general $y$-stable model, then only one direction holds.

Theorem 7 For any fuzzy formulas $F$ and $G$, if $I$ is a y-stable model of $F \otimes \neg G$ (relative to $\mathbf{p}$ ), then $I$ is a $y$-stable model of $F$ (relative to $\mathbf{p}$ ) and $I \models_{y} \neg G$.

Example 12. The other direction, that is, "if $I$ is a $y$-stable model of $F$ and $I \models_{y} \neg G$, then $I$ is a $y$-stable model of $F \otimes \neg G$," does not hold in general. For example, consider $F=G=p$ and $\otimes$ to be $\otimes_{l}$, and interpretation $I=\{(p, 0.4)\}$. Clearly $I$ is a 0.4-stable model of $p$ and $I \models_{0.4} \neg p$, but $I$ is not a 0.4 -stable model of $p \otimes_{l} \neg p$. In fact, $I$ is not even a 0.4 -model of the formula. 


\subsection{Theorem on Choice Formulas}

In the Boolean stable model semantics, formulas of the form $p \vee \neg p$ are called choice formulas, and adding them to the program makes atoms $p$ exempt from minimization. Choice formulas have been shown to be useful in composing a program in the "Generateand-Test" method. This section shows their counterpart in the fuzzy stable model semantics.

For any fuzzy atom $p$, Choice $(p)$ stands for $p \oplus_{l} \neg_{s} p$. For any list $\mathbf{p}=\left(p_{1}, \ldots p_{n}\right)$ of fuzzy atoms, Choice $(\mathbf{p})$ stands for Choice $\left(p_{1}\right) \otimes \ldots \otimes$ Choice $\left(p_{n}\right)$, where $\otimes$ is any fuzzy conjunction.

The following proposition tells that choice formulas are tautological.

Proposition 2 For any fuzzy interpretation I and any list $\mathbf{p}$ of fuzzy atoms, $I=_{1}$ Choice $(\mathbf{p})$.

Theorem 8 is an extension of Theorem 2 from [10].

Theorem $\mathbf{8}$ (a) If I is a y-stable model of $F$ relative to $\mathbf{p} \cup \mathbf{q}$, then $I$ is a $y$-stable model of $F$ relative to $\mathbf{p}$.

(b) $I$ is a 1-stable model of $F$ relative to $\mathbf{p}$ iff $I$ is a 1-stable model of $F \otimes$ Choice $(\mathbf{q})$ relative to $\mathbf{p} \cup \mathbf{q}$.

Theorem 8 (b) does not hold for arbitrary threshold $y$ (i.e., if " $1-$ " is replaced with " $y$-"). For example, consider $F=\neg s \neg s q$ and $I=\{(q, 0.5)\}$. Clearly $I$ is a 0.5 -model of $F$, and thus $I$ is a 0.5 -stable model of $F$ relative to $\emptyset$. However, $I$ is not a 0.5 -stable model of $F \otimes_{m}$ Choice $\left.(q)=\neg_{s}\right\urcorner_{s} q \otimes_{m}\left(q \oplus_{l} \neg_{s} q\right)$ relative to $\emptyset \cup\{q\}$, as witnessed by $J=\{(q, 0)\}$.

Since the 1-stable models of $F$ relative to $\emptyset$ are the models of $F$, it follows from Theorem 8 (b) that the 1-stable models of $F \otimes$ Choice $(\sigma)$ relative to $\sigma$ are exactly the 1-models of $F$.

Corollary 1 Let $F$ be a fuzzy formula of a finite signature $\sigma . I$ is a 1-model of $F$ iff $I$ is a 1-stable model of $F \otimes$ Choice $(\sigma)$ relative to $\sigma$.

Example 13. Consider the fuzzy formula $F=\neg_{s} p \rightarrow_{r} q$. Although any interpretation $I$ that satisfies $1-p^{I} \leq q^{I}$ is a 1 -model of $F$, among them only $\{(p, 0),(q, 1)\}$ is a 1 -stable model of $F$. However, we check that all 1-models of $F$ are exactly the 1-stable models of $G=F \otimes_{m}$ Choice $(p) \otimes_{m}$ Choice $(q): G^{*}(u, v)$ is

$$
\left(\neg_{s} p \rightarrow_{r} q\right) \otimes_{m}\left(\neg_{s} p \rightarrow_{r} v\right) \otimes_{m}\left(u \oplus_{l} \neg_{s} p\right) \otimes_{m}\left(v \oplus_{l} \neg_{s} q\right)
$$

and for $K=I \cup J_{u v}^{p q}$,

$$
G^{*}(u, v)^{K}=1 \otimes_{m}\left(\left(1-p^{K}\right) \rightarrow_{r} v^{K}\right) \otimes_{m}\left(u^{K} \oplus_{l}\left(1-p^{K}\right)\right) \otimes_{m}\left(v^{K} \oplus_{l}\left(1-q^{K}\right)\right) .
$$

So, for $K$ to satisfy $G^{*}(u, v)$ to degree $1, u^{K}$ should be at least $p^{K}$ and $v^{K}$ should be at least $q^{K}$. So there does not exist $J<^{p q} I$ such that $I \cup J_{u v}^{p q} \models_{1} G^{*}(u, v)$, from which it follows that $I$ is a 1 -stable model of $G$. 


\section{Other Related Work}

Several approaches to incorporating fuzziness into the answer set programming framework have been proposed. In this paper, we have formally compared our approach to [12] and [2]. Most of them consider the specific syntax where each formula is of the rule form $h \leftarrow B$ where $h$ is an atom and $B$ is a formula [4-7]. Among them, [4-6] allow $B$ to be any arbitrary formula corresponding to an increasing function whose arguments are the atoms appearing in the formula. [7] allows $B$ to correspond to either an increasing function or a decreasing function. [9] considers the normal program syntax, i.e., each rule is of the form $l_{0} \leftarrow l_{1} \otimes \ldots \otimes l_{m} \otimes n o t l_{m+1} \otimes \ldots \otimes n o t l_{n}$, where each $l_{i}$ is an atom or the strong negation of an atom. In terms of semantics, most of the previous works rely on the notion of immediate consequence operator and relate the fixpoint of this operator to the minimal model of a positive program. ${ }^{7}$ Similar to the approach [2] has adopted, the answer set of a positive program is defined as its minimal model, while an answer set of a non-positive program is defined in terms of the minimal model of the reduct, which is a positive program obtained based on the normal program and the specific interpretation being checked. [8] has proposed a semantics based on the notion of an unfounded set.

It is worth noting that some of the related works have discussed the so-called residuated programs [4-6,9], where each rule $h \leftarrow B$ is assigned a weight $\theta$, and a rule is satisfied by an interpretation $I$ if $I(h \leftarrow B) \geq \theta$. According to [5], this class of programs is able to capture many other logic programming paradigms, such as possibilistic logic programming, hybrid probabilistic logic programming, generalized annotated logic programming. Furthermore, as shown in [5], a weighted rule $(h \leftarrow B, \theta)$ can be simulated by $h \leftarrow B \otimes \theta$, where $(\otimes, \leftarrow)$ forms an "adjoint pair."

It is well known in the Boolean stable model semantics that strong negation can be represented in terms of new atoms [10]. Our adaptation in the fuzzy stable model semantics is similar to the method from [9], in which the consistency of an interpretation is guaranteed by imposing the extra restriction $I(\sim p) \leq \sim I(p)$ for all atom $p$. Strong negation and consistency have also been studied in $[13,14]$.

\section{Conclusion}

We introduced a stable model semantics for fuzzy propositional formulas, which generalizes both the Boolean stable model semantics and fuzzy propositional logic. The syntax is the same as the syntax of fuzzy propositional logic, but the semantics defines stable models instead of models. The formalism allows highly configurable default reasoning involving fuzzy truth values. Our semantics, when we restrict threshold to be 1 and assume all atoms to be subject to minimization, is essentially equivalent to fuzzy equilibrium logic, but is much simpler. To the best of our knowledge, our representation of the commonsense law of inertia involving fuzzy values is new. The representation uses nested fuzzy operators, which are not available in other fuzzy ASP semantics for a restricted syntax.

We showed that several traditional results in answer set programming can be naturally extended to this formalism, and expect that more results can be carried over. Future work includes implementing this language using mixed integer programming solvers or bilevel programming solvers [15].

\footnotetext{
${ }^{7}$ We call a program positive if it does not contain any default negation.
} 
Acknowledgements We are grateful to Joseph Babb, Michael Bartholomew, Enrico Marchioni, and the anonymous referees for their useful comments and discussions related to this paper. This work was partially supported by the National Science Foundation under Grant IIS-1319794 and by the South Korea IT R\&D program MKE/KIAT 2010TD-300404-001.

\section{References}

1. Lifschitz, V.: What is answer set programming? In: Proceedings of the AAAI Conference on Artificial Intelligence, MIT Press (2008) 1594-1597

2. Lukasiewicz, T.: Fuzzy description logic programs under the answer set semantics for the semantic web. In Eiter, T., Franconi, E., Hodgson, R., Stephens, S., eds.: RuleML, IEEE Computer Society (2006) 89-96

3. Janssen, J., Vermeir, D., Schockaert, S., Cock, M.D.: Reducing fuzzy answer set programming to model finding in fuzzy logics. TPLP 12(6) (2012) 811-842

4. Vojtás, P.: Fuzzy logic programming. Fuzzy Sets and Systems 124(3) (2001) 361-370

5. Damásio, C.V., Pereira, L.M.: Monotonic and residuated logic programs. In Benferhat, S., Besnard, P., eds.: ECSQARU. Volume 2143 of Lecture Notes in Computer Science., Springer (2001) 748-759

6. Medina, J., Ojeda-Aciego, M., Vojtás, P.: Multi-adjoint logic programming with continuous semantics. In: Proceedings of International Conference on Logic Programming and Nonmonotonic Reasoning (LPNMR). (2001) 351-364

7. Damásio, C.V., Pereira, L.M.: Antitonic logic programs. In: Proceedings of International Conference on Logic Programming and Nonmonotonic Reasoning (LPNMR). (2001) 379392

8. Nieuwenborgh, D.V., Cock, M.D., Vermeir, D.: An introduction to fuzzy answer set programming. Ann. Math. Artif. Intell. 50(3-4) (2007) 363-388

9. Madrid, N., Ojeda-Aciego, M.: Towards a fuzzy answer set semantics for residuated logic programs. In: Web Intelligence/IAT Workshops, IEEE (2008) 260-264

10. Ferraris, P., Lee, J., Lifschitz, V.: Stable models and circumscription. Artificial Intelligence 175 (2011) 236-263

11. Hajek, P.: Mathematics of Fuzzy Logic. Kluwer (1998)

12. Schockaert, S., Janssen, J., Vermeir, D.: Fuzzy equilibrium logic: Declarative problem solving in continuous domains. ACM Trans. Comput. Log. 13(4) (2012) 33

13. Madrid, N., Ojeda-Aciego, M.: Measuring inconsistency in fuzzy answer set semantics. IEEE T. Fuzzy Systems 19(4) (2011) 605-622

14. Madrid, N., Ojeda-Aciego, M.: On coherence and consistence in fuzzy answer set semantics for residuated logic programs. In Gesù, V.D., Pal, S.K., Petrosino, A., eds.: WILF. Volume 5571 of Lecture Notes in Computer Science., Springer (2009) 60-67

15. Alviano, M., Peñaloza, R.: Fuzzy answer sets approximations. TPLP 13(4-5) (2013) 753-767 